\title{
Disorder and Diffusion in Mayenite
}

\author{
H. Boysen ${ }^{a}, *$ I. KAiser-BischofF ${ }^{a}$, M. LerCh $^{b}$, S. Berendts $^{b}$, M. Hoelzel $^{c}$ \\ AND A. SENYSHYN ${ }^{c}$ \\ ${ }^{a}$ Department für Geo- und Umweltwissenschaften, Sektion Kristallographie \\ LMU München, Am Coulombwall 1, 85748 Garching, Germany \\ ${ }^{b}$ Institut für Chemie, TU Berlin, Str. des 17. Juni 135, 10623 Berlin, Germany \\ ${ }^{c}$ TU Darmstadt, Institute of Materials Science, Petersenstr. 23, 64287 Darmstadt, Germany
}

\begin{abstract}
Mayenite, $\mathrm{Ca}_{12} \mathrm{Al}_{14} \mathrm{O}_{33}$, has attracted enormous attention for novel technological applications after the discovery of its high oxygen ionic conductivity. The crystal structure consists of a calcium-aluminate framework, comprising 32 of the 33 oxygen anions. The remaining oxygen is distributed over $1 / 6$ of large cages within the framework. The true structure is heavily disordered and usually non-stoichiometric due to the presence of extra anions and is presented for four samples: pure oxygen mayenite (O-mayenite), partly $(\mathrm{O} / \mathrm{N}$-mayenite) and fully (N-mayenite) exchanged by nitrogen and doped with iron (Fe-mayenite). All samples were investigated by neutron powder diffraction up to $1050^{\circ} \mathrm{C}$. Data were analysed by the Rietveld method and by difference Fourier methods. As prepared O-mayenite contains $\mathrm{O}_{2}^{-}$and $\mathrm{OH}^{-}, \mathrm{N}-$ and $\mathrm{O} / \mathrm{N}$-mayenite also $\mathrm{NH}_{2}^{-}, \mathrm{NH}^{2-}$ and $\mathrm{N}^{3-}$, while Fe-mayenite was free of extra radicals. In $\mathrm{O}$ - and $\mathrm{N}$-mayenite the extra species are lost under vacuum conditions above $c a .700{ }^{\circ} \mathrm{C}$, whereas $\mathrm{O} / \mathrm{N}$-mayenite retained $\mathrm{NH}_{2}^{-}$up to high temperatures. Diffusion of oxygen proceeds via a jump-like interstitialcy process involving exchange of the "free" oxygen with framework oxygen, coupled to relaxations of $\mathrm{Ca}$ ions. In $\mathrm{O} / \mathrm{N}$-mayenite $\mathrm{NH}_{2}^{-}$diffuses via interstitial process. In Fe-mayenite encaged oxygen is "invisible" due to extreme delocalisation or loss.
\end{abstract}

PACS numbers: 61.43.-j, 61.66.-f, 61.05.fm

\section{Introduction}

Mayenite has long been known as a major component of calcium aluminate cements. Its crystal structure and composition (ideally: $\mathrm{Ca}_{12} \mathrm{Al}_{14} \mathrm{O}_{33}$ ) have first been determined by Büssem and Eitel in 1936 [1]. In cement industry it is often abbreviated as $\mathrm{C} 12 \mathrm{~A} 7$ derived from the formula $12 \mathrm{CaO} \cdot 7 \mathrm{Al}_{2} \mathrm{O}_{3}$. It was discovered as a mineral in 1964 near Mayen (Germany) from which it got its name [2]. After the discovery of its high oxide ion conductivity in 1988 [3], only one order of magnitude lower than that of yttria-stabilized zirconia (YSZ), it has recently attracted enormous attention for novel technological applications such as transparent conductive oxide, catalyst for the combustion of volatile organic compounds, oxygen ionic conductor, ion emitter, etc.

These exciting properties are related to its peculiar crystal structure. In simple terms it may be described as a framework of corner connected $\mathrm{AlO}_{4}$-tetrahedra with some linkages bridged by Ca. This framework comprises 32 of the 33 oxygen atoms; the "extra" one is distributed at random in $1 / 6$ of large "cages" within the framework (Fig. 1). These cages are connected via large "windows" (shaded area in Fig. 1) and an almost "free" diffusion of oxygen seems readily conceivable. This simple view

\footnotetext{
* corresponding author; e-mail: boysen@lmu.de
}

would also explain the fact that $\mathrm{O}^{2-}$ can easily be exchanged by other anions like $\mathrm{F}^{-}, \mathrm{Cl}^{-}, \mathrm{OH}^{-}, \mathrm{S}^{2-}$, etc., and even electrons $\mathrm{e}^{-}$forming an electride. As a new approach we succeeded in substituting $\mathrm{N}^{3-}$ for $\mathrm{O}^{2-}$ with the aim to produce a pure nitrogen conductor. First measurements by J. Janek (University of Giessen/Germany; private communication) showed that the electrical conductivity of a partly exchanged $\mathrm{O} / \mathrm{N}$-mayenite has much higher activation energy $(2.19 \mathrm{eV})$ than that of pure O-mayenite $(0.91 \mathrm{eV})$. As a consequence the absolute conductivity exceeds that of YSZ above $650^{\circ} \mathrm{C}$.

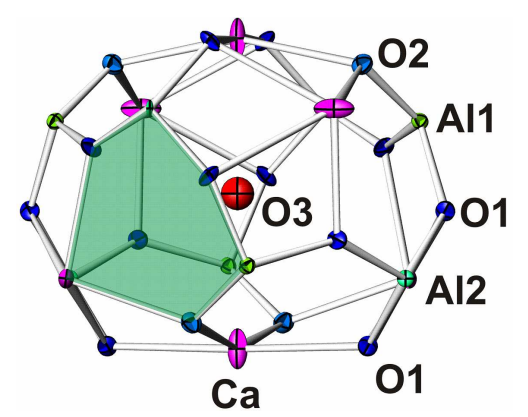

Fig. 1. Illustration of a cage within the framework structure of mayenite. Atomic displacement ellipsoids refer to O-mayenite at $T=1050^{\circ} \mathrm{C}[4]$. The shaded area shows a "window" connecting two adjacent cages formed by a six-atom ring (Ca-O1-Al2-O1-Al1-O2). 
The renewed interest in mayenite stimulated various new structure determinations, both experimentally (e.g. [4-7]) and theoretically (e.g. [8]). They have revealed that the above view of the crystal structure and diffusion process is certainly too simple. The structure is strongly disordered. This may e.g. be appreciated from the atomic displacement ellipsoids shown in Fig. 1 (after [4]): those of $\mathrm{Ca}$ are strongly elongated along the $\overline{4}$ axis running vertically through the cage and the very large extension of the encaged $\mathrm{O}$. This reflects the most prominent feature, namely that $\mathrm{Ca}$ atoms are displaced into the interior of the cage, if, and only if this is occupied by an oxygen ion. Further cage wall distortions are described in the cited references.

In this paper we present a comparison of pure oxygen mayenite (O-mayenite in the following) with fully (N-mayenite) and partly $(\mathrm{O} / \mathrm{N}$-mayenite) exchanged nitrogen mayenite. In addition, some results are included for a sample doped with $\mathrm{Fe}$ on the cation site (Fe-mayenite).

\section{Experimental}

Pure O-mayenite powder samples were synthesized by a solid state route [4]. Nitrogen exchanged samples were obtained by ammonolysis of these samples [9]. The iron doped sample was synthesized via a sol-gel route [10]. From chemical nitrogen analysis and the refined occupancies from the fits to the neutron powder diffraction data the following compositions were obtained: O-mayenite $\mathrm{Ca}_{12} \mathrm{Al}_{14} \mathrm{O}_{32} \mathrm{O}_{1.57}$, N-mayenite $\mathrm{Ca}_{12} \mathrm{Al}_{14} \mathrm{O}_{32} \mathrm{~N}_{1.27}$, O/N-mayenite $\mathrm{Ca}_{12} \mathrm{Al}_{14} \mathrm{O}_{32} \mathrm{~N}_{0.5} \mathrm{O}_{0.6}$ and Fe-mayenite $\mathrm{Ca}_{12} \mathrm{Al}_{13.75} \mathrm{Fe}_{0.25} \mathrm{O}_{32} \mathrm{O}_{1}$. All samples except Fe-mayenite contained varying amounts of hydrogen (not respected in the above given compositions) as revealed by the incoherent background in the neutron powder patterns. Neutron powder experiments were carried out at the diffractometer SPODI at the FRM2 in Garching [11] at various temperatures up to $1050^{\circ} \mathrm{C}$ using a wavelength of $1.548 \AA$ and a high-temperature niobium vacuum furnace. The data were analysed by the Rietveld method including anharmonic Debye-Waller factors and by difference Fourier methods with JANA2000 [12]. All samples contained small amounts of side phases [10] which are neglected here.

\section{Disorder}

The chemical compositions quoted above mean that all samples except Fe-mayenite exhibit considerable excess occupancy over the stoichiometric values. This is most pronounced for $\mathrm{N}$-mayenite for which the expected $\mathrm{N}$ content would be 0.67 according to the substitution of $1 \mathrm{O}^{2-}$ by $2 / 3 \mathrm{~N}^{3-}$. This is explained by the inclusion of other anions like $\mathrm{O}_{2}^{-}, \mathrm{O}_{2}^{2-}, \mathrm{O}^{-}$and/or $\mathrm{OH}^{-}$in $\mathrm{O}$-mayenite and $\mathrm{N}_{2}^{2-}, \mathrm{NH}_{2}^{-}$and/or $\mathrm{NH}^{2-}$ in N-mayenite. Of these $\mathrm{OH}^{-}$ and $\mathrm{O}_{2}^{-}$could be substantiated for O-mayenite [4], $\mathrm{NH}_{2}^{-}$ for $\mathrm{N}$-mayenite [10] by difference Fourier methods.
Figure 2 shows the temperature dependence of the refined occupancies for all samples. The horizontal lines indicate the occupancies for ideal stoichiometric compositions. It should be noted that those for $\mathrm{N}$ and $\mathrm{O}$ are practically identical due to the accidental fact that the neutron scattering lengths $b_{\mathrm{N}} \approx(3 / 2) b_{\mathrm{O}}$. Both $\mathrm{O}$ - and $\mathrm{N}$-mayenite become stoichiometric around $700{ }^{\circ} \mathrm{C}$ due to the loss of hydrogen-containing species (as proven by the reduction of the incoherent background in the neutron powder patterns) and the dissolution of $\mathrm{O}_{2}^{-}$in the case of O-mayenite. In contrast, $\mathrm{O} / \mathrm{N}$-mayenite retains its excess occupancy up to high temperatures. However, there is a considerable decrease of the incoherent background due to $\mathrm{H}$ indicating the loss of some but not all hydrogen. It can be argued that only $\mathrm{OH}^{-}$is transformed to $\mathrm{O}^{2-}$ while $\mathrm{NH}_{2}^{-}$is still present at high temperatures. The stoichiometric occupancy of Fe-mayenite does not change with temperature, but it should be noted that there seems to be some reduction already at $700{ }^{\circ} \mathrm{C}$ and a very large error bar at $900^{\circ} \mathrm{C}$ makes this latter value rather unreliable (see below).

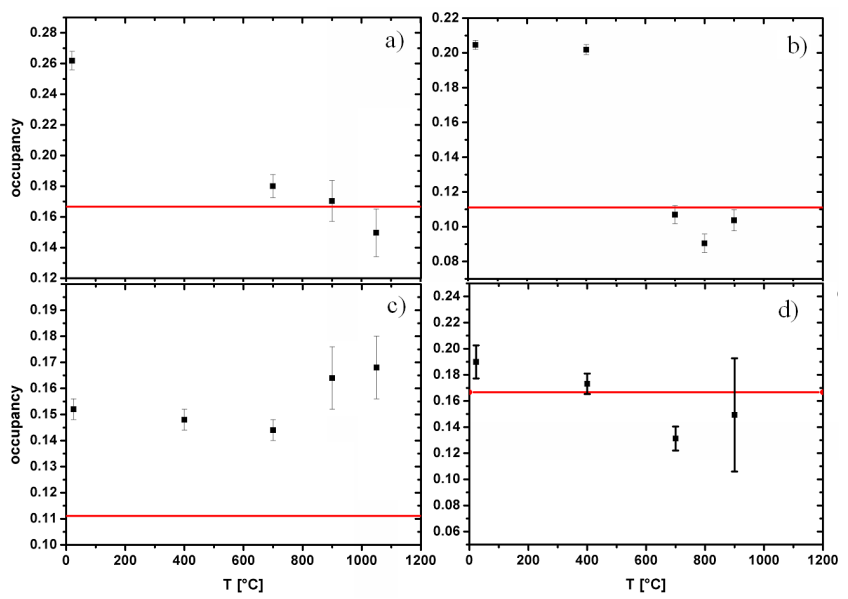

Fig. 2. Temperature dependence of occupancies for $\mathrm{O}$-mayenite (a), N-mayenite (b), O/N-mayenite (c) and Fe-mayenite (d).

\section{Diffusion}

Figure 3 compares the temperature dependences of the isotropic atomic displacement parameters for all samples. Generally being "normal" at low temperatures they become exceptionally large at high temperatures, corresponding to root mean square (rms) amplitudes of ca. $0.5 \AA$ for $\mathrm{O}$ - and $\mathrm{N}$-mayenite and $c a .0 .7 \AA$ for $\mathrm{O} / \mathrm{N}$ and Fe-mayenite. These values indicate extreme disorder of the encaged species possibly related to their diffusion properties. It should, however, again be noted that the error bar for Fe-mayenite is extremely large and therefore unreliable.

Further insight into the diffusion properties can be gained from the high temperature difference Fourier plots 


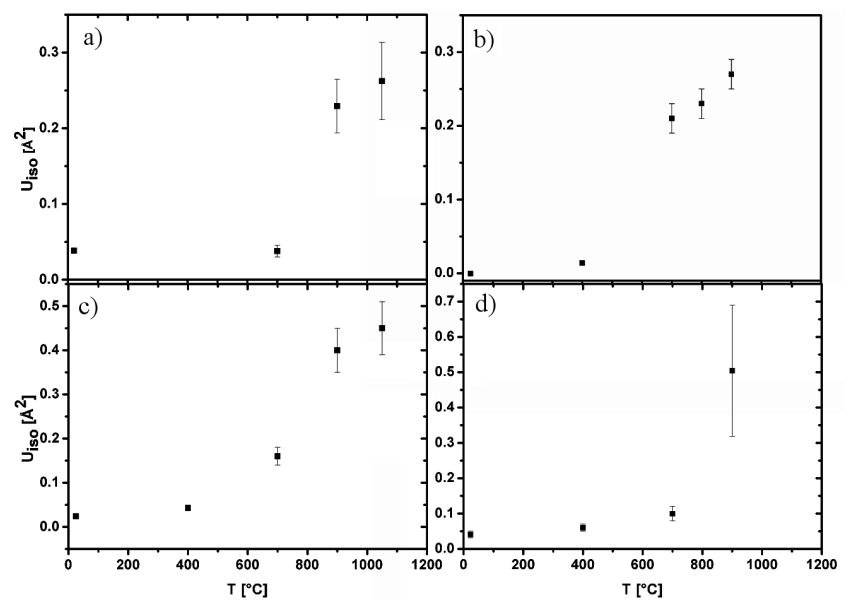

Fig. 3. Temperature dependence of isotropic displacement parameters of encaged anions for O-mayenite (a), $\mathrm{N}$-mayenite (b), O/N-mayenite (c) and Fe-mayenite (d).

presented in Fig. 4. In O-mayenite (Fig. 4a) oxygen density maxima are on off-centre positions (4-fold split-positions due to the local $\overline{4}$ symmetry). In addition, $\mathrm{Ca}$ is also located sideward from the central $\overline{4}$-axis. The crosswise distances between the corresponding $\mathrm{Ca}$ and $\mathrm{O}$ peaks are $c a .2 .4 \AA$, i.e. they form bond-like $\mathrm{Ca}-\mathrm{O}$ pairs. Hence O is not really "loosely bound" or "free" as often termed in the elder literature and we have to envisage a coupled $\mathrm{Ca}-\mathrm{O}$ diffusion process. Further difference Fourier plots and test refinements including anharmonic terms in the Debye-Waller factor [13] did not show any continuous density between adjacent cages as would be expected for a "normal" interstitial diffusion process. Hence diffusion proceeds via exchange with framework $\mathrm{O}$, i.e. an interstitialcy process, in excellent agreement with theoretical calculations [8].

N-mayenite (Fig. 4b) has a maximum density in the centre of the cage, though elongated along the larger extension of the cage. Again no continuous density between the cages was found, seemingly excluding interstitial diffusion. On the other hand, no nitrogen was found within the framework, seemingly excluding interstitialcy processes. Unfortunately, no macroscopic measurements of the diffusion properties of this sample are available so far. It may, however, be argued that the very high negative charge of $\mathrm{N}^{3-}$ may impede easy diffusion and the observed large smearing of the density is simply due to heavy static and/or dynamic disorder.

$\mathrm{O} / \mathrm{N}$-mayenite (Fig. 4c) shows a triple peaked density which may be envisaged as a superposition of that of $\mathrm{O}-$ and $\mathrm{N}$-mayenite, i.e. the side peaks represent $\mathrm{O}$ and the middle peak $\mathrm{N}$. In this case continuous density between the cages could be identified. Assuming jump diffusion of $\mathrm{O}$ as before and remembering that the sample still contains $\mathrm{NH}_{2}^{-}$it may be concluded that $\mathrm{NH}_{2}^{-}$migrates via interstitial diffusion process [9].

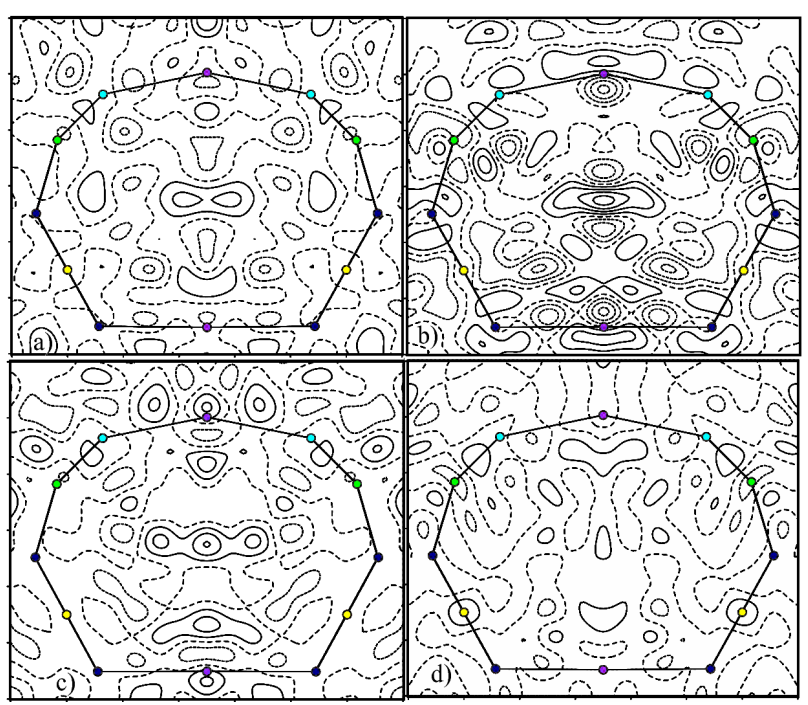

Fig. 4. High temperature difference Fourier maps through the centre of the cage after refinement with framework atoms only for O-mayenite (a), N-mayenite (b), O/N-mayenite (c) and Fe-mayenite (d).

No unambiguous residual density can be discerned in Fe-mayenite (Fig. 4d), therewith explaining the large errors of the corresponding occupancy and atomic displacement parameters. This "invisibility" of any oxygen density within the cage may be due to even more extreme delocalization (extremely high diffusion) or to a loss of oxygen in conjunction with Fe reduction. Further work is needed to decide between these and possibly other explanations.

\section{Conclusions}

As prepared (solid-state route) O-mayenite contains extra anions, of which $\mathrm{OH}^{-}$and $\mathrm{O}_{2}^{-}$were substantiated. These species are (irreversibly) lost at high temperatures under vacuum conditions. Diffusion proceeds via exchange with framework oxygen (intersticialcy process) and is coupled to relaxations of the Ca ions. N-mayenite prepared via ammonolysis contains extra anions, of which $\mathrm{NH}_{2}^{-}$was substantiated. They are (irreversibly) lost at high temperatures under vacuum conditions. The diffusion properties remain unclear and the existence of a pure nitrogen conductor is still to be verified. Surprisingly, in $\mathrm{O} / \mathrm{N}$-mayenite $\mathrm{NH}_{2}^{-}$species are not lost at high temperatures under the same vacuum conditions. The reason for this is not clear at the moment. Diffusion of $\mathrm{O}$ is via the interstitialcy mechanism, while that of $\mathrm{N}$ proceeds as $\mathrm{NH}_{2}^{-}$via an interstitial mechanism. This may be related to its lower charge allowing easier diffusion. Fe-mayenite prepared via sol-gel route does not contain extra anions. It must be emphasized that this is not due to the different synthesis route, since a similarly prepared sample with a lower Fe content behaves just as the pure O-mayenite prepared via the solid state route. Oxygen 
density within the cage is either extremely delocalised or absent at high temperatures. More work is needed to tackle these issues.

\section{Acknowledgments}

This work forms part of a joint DFG project (SPP1136) and is supported under BO 1199/2 and LE 781/10.

\section{References}

[1] W. Büssem, A. Eitel, Z. Kristallogr. 95, 175 (1936).

[2] G.N. Hentschel, N. Jb. Miner. Mh. 22, (1964).

[3] M. Lacerda, J.T.S. Irvine, F.P. Glasser, A.R. West, Nature 332, 525 (1988).

[4] H. Boysen, M. Lerch, A. Stys, A. Senyshyn, Acta Crystallogr. B 63, 675 (2007).

[5] L. Palacios, A. De La Torres, S. Bruque, J. Garcia-Munoz, S. Garcia-Granda, D. Sheptyakov, M.A.G. Aranda, Inorg. Chem. 46, 4167 (2007).
[6] T. Nomura, K. Hayashi, Y. Kubota, T. Kamiya, M. Hirano, M. Takata, H. Hosono, Chem. Lett. 36, 902 (2007).

[7] R. Kiyanagi, J.W. Richardson, N. Sakamoto, M. Yoshimura, Solid State Ionics 179, 2365 (2008).

[8] P.V. Sushko, A.L. Shluger, K. Hayashi, M. Hirano, H. Hosono, Phys. Rev. B 73, 014101 (2006).

[9] H. Boysen, I. Kaiser-Bischoff, M. Lerch, Diffusion Fundamentals 8, 2 (2008).

[10] H. Boysen, I. Kaiser-Bischoff, M. Lerch, S. Berendts, A. Börger, D.M. Trots, M. Hoelzel, A. Senyshyn, Z. Kristallogr. S30, 323 (2009).

[11] M. Hoelzel, A. Senyshyn, R. Gilles, H. Boysen, H. Fuess, Neutron News 18, 23 (2007).

[12] V. Petricek, M. Dusek, L. Palatinus, Jana2000. The Crystallographic Computing System, Institute of Physics, Praha, Czech Republic 2000.

[13] H. Boysen, Z. Kristallogr. 218, 123 (2003). 